\title{
Los otros datos ausentes que también importan: estado de salud bucodental de la población chilena.
}

\section{Sr. Editor:}

Las enfermedades bucales continúan siendo un importante problema de Salud Pública en Chile y el mundo por su alta prevalencia, impacto negativo en múltiples dimensiones de salud, bienestar y calidad de vida, y por el alto costo de su tratamiento. Tal como lo reconoce la Organización Panamericana de la Salud (OPS), la salud bucodental es un aspecto crítico de las condiciones generales de salud en la Región de las Américas debido a su peso en la carga general de morbilidad y a la asociación de la salud bucodental con factores de riesgo de Enfermedades No Transmisibles $(E N T)^{1}$. En Chile, los datos epidemiológicos de salud bucodental no están actualizados y para la elaboración del Plan Nacional de Salud Bucal 2018-2030 se utilizó información obtenida de diversos estudios realizados entre 2007 y 2010 en las distintas etapas del ciclo vital, con metodologías diferentes y desarrollados en distintos subgrupos de la población ${ }^{2}$.

Algunas encuestas poblacionales como la Encuesta Nacional de Salud (ENS), la Encuesta Nacional de Calidad de Vida (ENCAVI) y la Encuesta Mundial de Salud Escolar (EMSE) incluyen preguntas relacionadas a salud bucodental y su asociación a calidad de vida. Sin embargo, ninguna ha incorporado el diagnóstico de salud bucodental poblacional realizado por profesional odontológico, la ENS es la única de ellas que ha logrado avanzar en este ámbito pues en sus versiones de 2003/2004 y 2016/2017 incorporó un diagnóstico de presencia de lesiones de caries cavitadas y de dentición no funcional (definida como la presencia de menos de 20 dientes en la cavidad oral) realizado por una profesional de enfermería. Creemos necesario que se incorpore a profesionales odontológicos en el diagnóstico bucal en estas encuestas poblacionales ya que son quienes desarrollan las competencias técnicas a lo largo de su formación para el diagnóstico correcto de las enfermedades bucodentales y, además, permitiría tener información que conlleve menos sesgos en los indicadores de salud bucodental. Otra ventaja es que se podría contar con más información de prevalencia de otras condiciones bucodentales y que no se limiten sólo a diagnosticar presencia/ausencia de lesiones cavitadas o número de dientes en boca, por ejemplo, a través del índice COPD o pérdida de inserción clínica para evaluar prevalencia de enfermedades periodontales. Sumado a ello, está el eventual aporte de estos estudios a la comparabilidad internacional de acuerdo a metodología propuesta por la $\mathrm{OMS}^{3}$.

El principio básico de la Epidemiología es que la distribución de las condiciones de salud en la población no ocurre al azar, sino que está relacionada a diversos determinantes biológicos, sociales, conductuales, etc. En el caso de las enfermedades de la cavidad oral está bien documentada la influencia de los determinantes sociales en el riesgo y prevalencia de estas patologías ${ }^{4}$, por lo que se hace imperioso contar con un sistema de vigilancia en salud bucodental que incluya indicadores que no sólo permitan estudiar la prevalencia/incidencia de enfermedad y se transformen en un simple conteo de casos, sino que también incorporen indicadores específicos de vulnerabilidad, como los determinantes sociales y conductuales asociados, por ejemplo, al índice significante de caries $^{5}$, para así estudiar las desigualdades injustas 
existentes y poder focalizar los escasos recursos en la población con mayor riesgo y mayor desventaja social. Tal como lo señala la Federación Dental Internacional en su Segunda Versión del Atlas de Salud Bucodental, la falta de información de calidad constituye una barrera para priorizar la prevención y tratamiento de las enfermedades bucodentales y limita el desarrollo de respuestas de salud pública eficaces ${ }^{6}$. En este mismo documento se expone la necesidad de incluir indicadores de salud y enfermedades bucodentales de forma sistemática que contengan datos sobre factores de riesgo, de mejorar los registros de cáncer oral, de mejorar el seguimiento de traumatismos orofaciales y malformaciones congénitas, y de contar con datos y registros asequibles con disponibilidad universal para la investigación y toma de decisiones políticas informadas.

La Estrategia Nacional de Salud en su Objetivo Estratégico 2 tiene incorporadas varias metas y Resultados Esperados (RE) en Salud Bucal, siendo el RE N 3 contar con un Sistema de información Epidemiológica y la "realización de un estudio poblacional de Salud Bucal en niños/as y adolescentes para evaluar las metas de impacto de la misma Estrategia Nacional de Salud"7, lo cual no ha sido realizado en los diez años que considera esta estrategia.

El Plan Nacional de Salud Bucal 2018-2030 (PNSB) desarrollado por el Departamento de Salud Bucal de la División de Prevención y Control de Enfermedades (DIPRECE) entre 2014 y 2017, en $\mathrm{Su}$ Eje Estratégico $\mathrm{N}^{\circ} 4$ Investigación, Monitoreo y Evaluación plantea como Objetivo Estratégico 10 "Instalar un sistema de vigilancia del estado de salud bucal de la población y su impacto en la calidad de vida" 2 . Para dar cumplimiento a dicho objetivo, en este documento se propone como Estrategia 10.2 "Actualizar el diagnóstico nacional de salud bucal" en las distintas etapas del ciclo vital mediante encuestas poblacionales realizadas por profesional odontológico, las cuales para salud bucal infantil y salud bucal del adolescente deberían estar cumplidas al año 2020 según la meta propuesta y en 2025 para adultos, sin que a la fecha se tenga información al respecto por parte del MINSAL.

La pandemia por COVID-19 ha puesto en jaque a los sistemas de salud de los países y en el caso de Chile no ha sido la excepción. El contexto actual ha manifestado la importancia de contar con sistemas de salud eficientes, de calidad y que cumplan su función esencial de protección a quienes se encuentren en mayor desventaja social o que requieran de mayores cuidados sanitarios. Esto sólo puede lograrse cuando las decisiones en políticas de salud se basan en información fidedigna y actualizada del estado de salud de la población, por lo que contar con datos epidemiológicos en salud bucodental permitirá un uso más costo-eficiente de los recursos destinados a los programas odontológicos actuales y los futuros que se vayan a implementar.

Recientemente se ha anunciado la creación de una Dirección Nacional de Odontología, siendo una oportunidad para asumir e implementar las estrategias pendientes para la vigilancia epidemiológica en salud bucodental en Chile. Consideramos que una de las primeras y fundamentales labores que deberá tener esta nueva institucionalidad es avanzar en disponer de datos epidemiológicos de mejor calidad y actualizados, acordes a los estándares propuestos por la OMS y que permitan comparabilidad internacional. En lo inmediato, sería interesante generar espacios de trabajo intersectorial que incluyan colaboración público-privada, desarrollo de fondos específicos de investigación en salud bucodental mediante mecanismos existentes como CORFO, CONICYT y FONIS, y se aproveche la territorialidad en que se sustenta la Atención Primaria de Salud para el levantamiento 
de información epidemiológica de calidad.

Sebastián Perry Pasten

Estudiante Especialidad en Salud Pública Odontológica, Facultad de Odontología, Universidad de Chile.

Correo: gperry@odontología.uchile.cl, sin conflictos de interés.

Marco Cornejo Ovalle

Profesor Asistente, Facultad de Odontología, Universidad de

Chile.

Correo: mcornejo@odontologia.uchile.cl,sin conflictos de interés.

\section{REFERENCIAS}

[1] PAHO. Proposed 10-Year Regional Plan on Oral Health for the Americas: Final Report. Provisional Agenda Item [Internet]. 2017;7(7):26-30. http://www1.paho.org/English/GOV/CD/ CD47-14-e.pdf

[2] Ministerio de Salud de Chile, Subsecretaría de Salud Pública, División de Control y Prevención de enfermedades $D$ de SB. Plan Nacional de Salud Bucal 2018-2030. 2017;80. https://www.minsal.cl/wpcontent/uploads/2017/12/PlanNacional-Salud-Bucal-2018-2030Consulta-Pública-20 12 2017.pdf

[3] OMS. Encuestas de Salud bucodental. Oms [Internet]. 1997;5(4):84. http://books.google.com/books?id=q21 Cqq7F9IMC\&pgis $=1$

[4] Arora A, Schwarz E, Blinkhorn AS tevenso. Risk factors for early childhood caries in disadvantaged populations. $\mathrm{J}$ Investig Clin Dent. 2011;2(4):223-8.

[5] Bratthall D. Introducing the significant caries index together with a proposal for a new global oral health goal for 12year-olds. Int Dent J. 2000;50(6):37884.

[6] FDI. El desafío de las enfermedades bucodentales. Una llamada a la acción global. [Internet]. Federación Dental Internacional. $2015 . \quad 14-6$ https://www.fdiworlddental.org/sites/def ault/files/media/documents/book sprea ds oh2 spanish.pdf

[7] Ministerio de Salud de Chile. Metas 2011-2020 Estrategia Nacional de Salud para el cumplimiento de los Objetivos
Sanitarios de la Década Para el cumplimiento de los 2011-2020 [Internet].

http://www.bibliotecaminsal.cl/wp/wpcontent/uploads/2011/12/Metas-20112020.pdf

\section{CÓMO CITAR ESTE ARTÍCULO}

Perry S, Cornejo M. Los otros datos ausentes que también importan: estado de salud bucodental de la población chilena. Appli. Sci. Dent. 2020; 1(3):4-6. DOI: 10.22370/asd.2020.1.3.2441 